Please do not remove this page

RMIT

UNIVERSITY

\title{
MPCVD processing of titanium-diffused LiNbO3 waveguides: optical characterisation and waveguide restoration
}

Trpkovski, Steven; Gibson, Brant; Bui, Lam; Huntington, S; Simpson, David; Ampem-Lassen, Eric; Rabeau, James

https://researchrepository.rmit.edu.au/esploro/outputs/9921862448901341/filesAndLinks?institution=61RMIT_INST\&index=null

Trpkovski, S., Gibson, B., Bui, L., Huntington, S., Simpson, D., Ampem-Lassen, E., Rabeau, J., \& Mitchell, A. (2006). MPCVD processing of titanium-diffused LiNbO3 waveguides: optical characterisation and waveguide restoration. Proceedings of the 2006 Conference on Optoelectronic and Microelectronic Materials and Devices (COMMAD 2006). https://doi.org/10.1109/COMMAD.2006.4429929

Published Version: https://doi.org/10.1109/COMMAD.2006.4429929

Repository homepage: https://researchrepository.rmit.edu.au (c) 2006 IEEE. Personal use of this material is permitted. However, permission to reprint/republish this material for advertising or promotional purposes or for creating new collective works for resale or redistribution to servers or lists, or to reuse any copyrighted component of this work in other works must be obtained from the IEEE.

Downloaded On 2023/04/26 17:59:32 +1000 


\title{
MPCVD Processing of Titanium-diffused $\mathrm{LiNbO}_{3}$ Waveguides: Optical Characterisation and Waveguide Restoration
}

\author{
S. Trpkovski*, L. A. Bui ${ }^{\dagger}$, B. C. Gibson*, S. T. Huntington*, D. Simpson*, E. Ampem-Lassen*, J. R. Rabeau*, \\ A. Mitchell ${ }^{\dagger}$ \\ * Quantum Communications Victoria, \\ School of Physics, The University of Melbourne, Parkville, Australia \\ Email: strp@unimelb.edu.au \\ $\uparrow$ School of Electrical and Computer Systems Engineering, \\ RMIT University, Melbourne, Australia \\ Email: lam.bui@rmit.edu.au
}

\begin{abstract}
This paper presents some initial findings that explore the material properties of $\mathrm{LiNbO}_{3}$ which has been exposed to a Microwave Plasma-enhanced Chemical Vapor Deposition (MPCVD) environment. The $\mathrm{LiNbO}_{3}$ was found to undergo a process known as 'reduction' when exposed to this environment. $A$ technique was developed to reverse this process and recover the $\mathrm{LiNbO}_{3}$, which is a crucial first step towards the integration of diamond-based single photon sources with $\mathrm{LiNbO}_{3}$ waveguide technologies.
\end{abstract}

Keywords- diamond; $\mathrm{MPCVD} ; \mathrm{LiNbO}_{3}$, waveguide, optical

\section{INTRODUCTION}

Titanium-diffused $\mathrm{LiNbO}_{3}$ waveguide integrated optic devices have become an industry standard for high-speed photonic information processing both in ultra-broadband digital communications and analogue defence applications. Integrated optics based on $\mathrm{LiNbO}_{3}$ also provides an excellent platform for scientific investigation of fundamental optical physics. Recently the deposition of nanocrystalline diamond directly on the end facet of optical fibers has been demonstrated, enabling the direct interfacing of diamond-based single photon sources with optical fiber systems [1]. This paper presents our recent efforts to realize nanocrystalline diamond on the end facets of $\mathrm{LiNbO}_{3}$ waveguide. The capability to integrate high-speed, low-loss $\mathrm{LiNbO}_{3}$ with single photon sources will create a flexible platform for practical research into quantum optics.

In this investigation we have exposed Titanium-diffused $\mathrm{LiNbO}_{3}$ waveguides to a Microwave Plasma-enhanced Chemical Vapor Deposition (MPCVD) environment; which is used to grow nanocrystalline diamond. The $\mathrm{LiNbO}_{3}$ transparency was significantly degraded during this process. Exposure to elevated temperatures is a common technique used for chemical reduction of $\mathrm{LiNbO}_{3}$, which improves surface acoustic wave device performance but on the downside, increases the optical attenuation of the material [2]. The motivation of this work requires the $\mathrm{LiNbO}_{3}$ to retain a low optical attenuation to enable propagation of very low levels of fluorescence. Therefore, a unique processing technique was investigated to reduce the optical attenuation after the $\mathrm{LiNbO}_{3}$ had suffered chemical reduction due to exposure to the MPCVD environment.

\section{SAMPLE PREPARATION AND CHARACTERISATION}

Both blank $\mathrm{LiNbO}_{3}$ and $\mathrm{Ti}$ diffused channel waveguide samples were investigated. The blank samples were directly obtained by dicing a 3 inch wafer into sizes of approximately $1 \times 2 \mathrm{~cm}$. The fabrication of channel waveguide samples comprised of a number of steps. The channel waveguides were fabricated using the whole wafer process. The fabrication commences with electron beam evaporation of $85 \mathrm{~nm}$ of Titanium, followed with photolithography of optical waveguides and finally completes with diffusion in wet $\mathrm{O}_{2}$ for 10 hours. The wafer was later diced into samples of size $1 \times 2 \mathrm{~cm}$ and the end facets of each sample were polished to optical quality for optical characterisation.

Prior to the MPCVD treatment, the $\mathrm{LiNbO}_{3}$ waveguide samples were characterised for optical losses at $1550 \mathrm{~nm}$. Since $\mathrm{LiNbO}_{3}$ is a birefringence crystal, the material loss and refractive indices are dependent on the light's polarization state[3]. For $\mathrm{LiNbO}_{3}$, the two possible polarization states are the parallel and the orthogonal polarization states. These polarization states are parallel and perpendicular to the optic axis of the crystal and therefore experience extra-ordinary and ordinary indices, respectively. Since the crystal properties are different for each polarization state, it is important to optically characterize for both polarizations.

A common technique used to measure waveguide loss utilizes the optical response obtained from the Fabry-Perot cavity formed between the waveguide two facets. This technique is commonly referred to as the Fabry-Perot (F-P) technique [4]. In this investigation, the need for accurate loss measurement and the ability to characterize for both ordinary and extraordinary polarization make the F-P technique most suitable. 


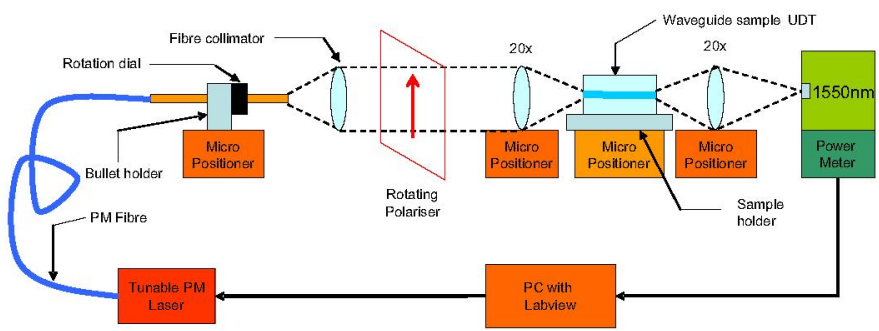

Figure 1: Experimental setup to characterize waveguide loss using the Fabry-Perot technique.

The experimental setup for measuring waveguide loss using F-P technique is outlined in Figure 1. A tunable laser was employed to vary the optical wavelength in the range between $1550.0 \mathrm{~nm}$ and $1550.2 \mathrm{~nm}$ with the scanning resolution of $1 \mathrm{pm}$. Figure 2 shows the transmission intensity through the waveguide as a function of wavelength for both polarization states.

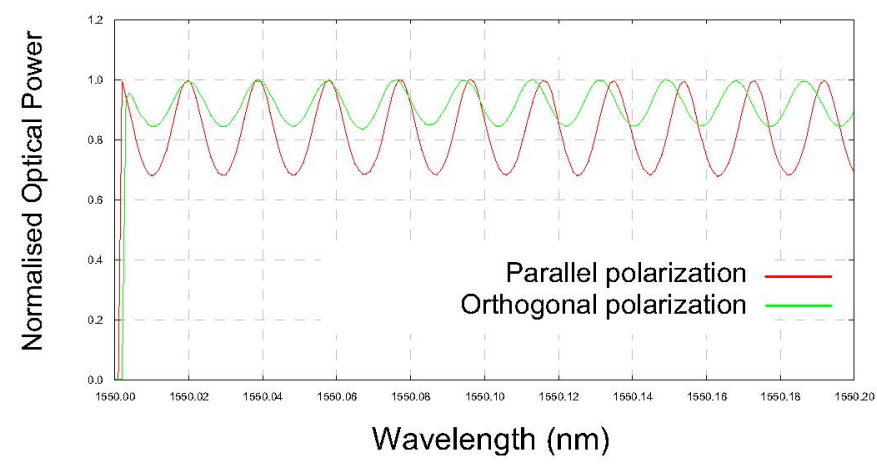

Figure 2: Measured Fabry Perot responses of the channel waveguides for parallel and perpendicular polarizations.

Assuming the theoretical indices of $\mathrm{LiNbO}_{3}$ for parallel and orthogonal polarizations to be 2.138 and 2.211 at $1550 \mathrm{~nm}$, respectively [3], the waveguide loss could be computed from the fringe contrast of the measured F-P response of Figure 2. These were found to be 1.37 and $5.25 \mathrm{~dB}$ for parallel and orthogonal polarizations, respectively, which are typical values for Titanium diffused $\mathrm{LiNbO}_{3}$ waveguides. It was anticipated that these results would enable direct comparison of the optical loss of the initial sample and the loss of the same sample after the reduction and then the regeneration.

\section{MPCVD PROCESSING OF LITHIUM NIOBATE SAMPLES}

The Titanium-diffused $\mathrm{LiNbO}_{3}$ waveguides were placed in a MPCVD reactor which consisted of a standard bell-jar growth chamber with a $1.2 \mathrm{~kW}$ microwave power supply. The waveguides were heated to $800{ }^{\circ} \mathrm{C}$ under 30 Torr, with gas mixtures of Hydrogenated Methane for 30 minutes. These conditions are similar to those used when growing nanocrystalline diamond on particular substrates, e.g. silica waveguides. It has previously been reported that when $\mathrm{LiNbO}_{3}$ is exposed to elevated temperatures $>200^{\circ} \mathrm{C}$ in an oxygen deficient atmosphere, the process of reduction occurs [5]. These conditions caused the $\mathrm{LiNbO}_{3}$ sample to transform into an opaque, black colored material due to increasing optical absorption, as illustrated in Figure $3 \mathrm{a}$.

It was assumed that the mixture of hydrogenated Methane could alter the reduction process due to the presence of Hydrogen gas. Investigative comparisons were performed between the basic reduction in vacuum (i.e. reduced in vacuum at 30 Torr at $800^{\circ} \mathrm{C}$ for 30 minutes) and the reduction in the MPCVD environment. After reduction, the samples appeared black with uniform texture. No damage to the polished surface was observed. Visual inspection of the reduced samples after the MPCVD treatment with a microscope could not distinguish the samples treated with and without the MPCVD environment. Figure 3 presents a sample after exposure to the MPCVD environment (Figure 3a) and an untreated $\mathrm{LiNbO}_{3}$ sample cutting from the same wafer for comparison (Figure $3 b)$.

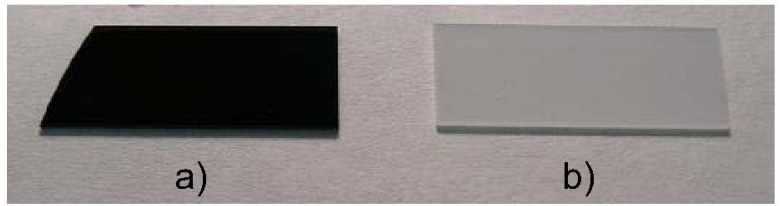

Figure 3: Reduced and untreated Lithium Niobate a) sample after reduction in gas and with the plasma b) Untreated sample. Sample reduced in vacuum is not shown.

Together with the blank $\mathrm{LiNbO}_{3}$ samples, a channel waveguide sample was also reduced in the MPCVD with the process gases and the plasma. Similar to the blank samples, the waveguide sample after reduction was black in color and no visual damage to the polished surface was observed.

\section{SAMPLE RESTORATION}

The increased optical loss of $\mathrm{LiNbO}_{3}$ after reduction is related to oxygen deficiency [5]. By annealing the reduced $\mathrm{LiNbO}_{3}$ in an oxygen atmosphere, it was believed that the reduction process could be reversed. We first experimented by annealing a $\mathrm{LiNbO}_{3}$ sample, which was reduced in vacuum without the MPCVD environment, in a wet $\mathrm{O}_{2}$ environment. The flow rate of $\mathrm{O}_{2}$ was $1 \mathrm{LPM}$ at $800^{\circ} \mathrm{C}$ over a time duration of 1 hour, which also allowed 15 minutes for the sample to heat up to $800^{\circ} \mathrm{C}$ and 15 minutes for it to cool down to room temperature. Figure $4 \mathrm{a}$ and $4 \mathrm{~b}$ show the untreated $\mathrm{LiNbO}_{3}$ sample and the reduced sample cut from the same wafer, respectively. Visual inspection of the regenerated sample showed the samples had the same transparency, color and surface quality as the untreated $\mathrm{LiNbO}_{3}$. This result suggests that the sample had been fully restored to its original quality.

Having obtained positive results with the samples that were reduced only in vacuum, a blank sample reduced in the 
MPCVD environment was regenerated using the same conditions. Figure $4 \mathrm{c}$ shows this sample together with the original untreated $\mathrm{LiNbO}_{3}$ sample. The $\mathrm{LiNbO}_{3}$ sample shown in Figure 4c exhibited a milky color and less transparency compared to its original condition. Unlike samples $4 a$ and $b$, formation of a surface film was obvious on the sample shown in Figure 4c. This film was partially combusted and presented some damage to the sample surface. Further examination of the sample under the microscope showed that the surface roughness degraded in areas where the film had been removed.

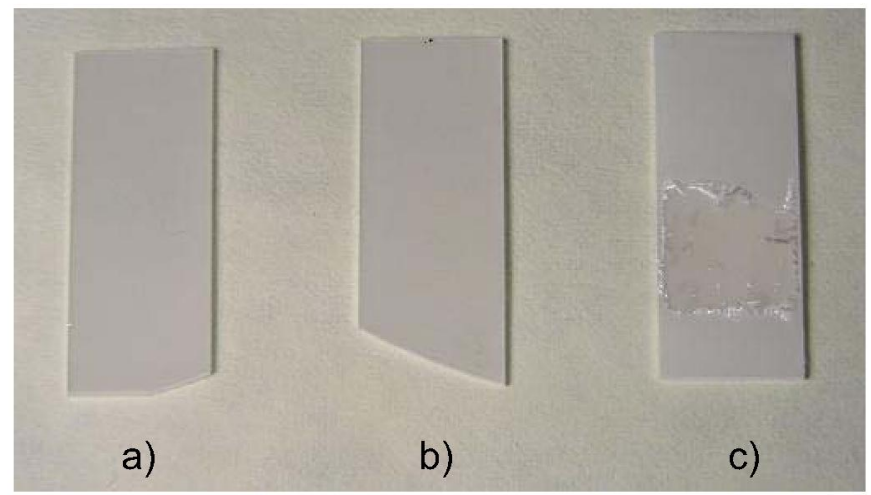

Figure 4: Untreated and regenerated samples reduced at various conditions a) Untreated Lithium Niobate b) sample reduced in vacuum and c) sample reduced in gas and with the plasma.

The reduced Titanium diffused $\mathrm{LiNbO}_{3}$ waveguide samples were also regenerated in similar conditions. Figures $5 \mathrm{a}$ and $\mathrm{b}$ show the channel waveguide prior to reduction and after regeneration, respectively. The surface roughness and coloration of the sample of Figure $5 b$ was found to be similar to that of Figure 4c, except where the channel waveguides had been defined. Formation of a surface thin-film was also evident. There were several locations of combustion of this film exposing the substrate underneath. The absence of the film in regions of the channel guides suggested that its formation was suppressed in the presence of Titanium. Optical guiding characteristic of the channel waveguides were tested by fiber

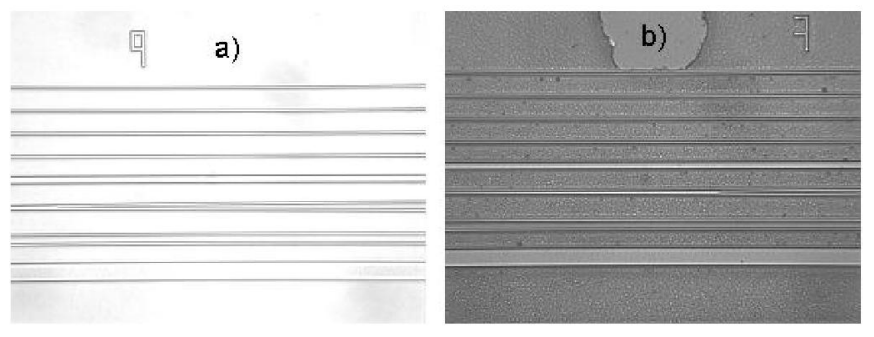

waveguide butt coupling and measurement of mode profile with a CCD camera. It was found that the waveguides no longer supported the parallel polarization mode but weakly guided the orthogonal polarization mode. Evidence of slab guiding by the surface thin film was also noticeable.

\section{DISCUSSION}

The relationship between $\mathrm{LiNbO}_{3}$ reduction and Oxygen deficiency has previously been discussed. We have shown that $\mathrm{LiNbO}_{3}$ could be recovered by an annealing process in an Oxygen rich environment. We have also found that $\mathrm{LiNbO}_{3}$ reduced in an MPCVD environment, couldn't be recovered to the same quality using the particular annealing process. We suggest that this is attributed to the molecules in the MPCVD environment; Hydrogen in this case combined with the crystal may have formed a different material to $\mathrm{LiNbO}_{3}$. The presence of such a material was likely to be near to the crystal surface and was evident as a thin-film observed in Section IV. This thin-film modified the optical properties of the waveguide as the propagating light leaked from the Ti-diffused core. Future work will focus on suppressing the formation of thin-film, which will improve the optical guiding properties.

\section{CONCLUSIONS}

Titanium-diffused $\mathrm{LiNbO}_{3}$ waveguides were exposed to a MPCVD environment, which had the effect of modifying the optical properties of the device. A technique, that involved an anneal in a wet oxygen atmosphere, was implemented to recover the reduced $\mathrm{LiNbO}_{3}$ waveguides. The preliminary results suggest that $\mathrm{LiNbO}_{3}$ waveguides can be exposed to a MPCVD environment whilst retaining their material properties. This is a crucial first step to enable the integration of diamondbased single photon sources with $\mathrm{LiNbO}_{3}$ waveguide technologies.

\section{REFERENCES}

E. M. Standifer, D. H. Jundt, R. G. Norwood, and P. F. Bordui, "Chemically reduced lithium niobate single crystals: processing, properties and improvements in SAW device fabrication and performance," presented at Frequency Control Symposium, 1998. Proceedings of the 1998 IEEE International, 1998.

K. K. Wong, Properties of Lithium Niobate: Institution of Engineering and Technology, 2002.

R. J. Deri and E. Kapon, "Low-loss III-V semiconductor optical waveguides," Quantum Electronics, IEEE Journal of, vol. 27, pp. 626-640, 1991.

L. Arizmendi, J. M. Cabrera, and F. Agullo-Lopez, "Defects induced in pure and doped LiNbO, by irradiation and thermal reduction," J. Phys. C: Solid State Phys., vol. 17, pp. 515-529, 1984.

Figure 5: Pictures of channel waveguides a) prior to reduction and $b$ ) after regeneration. 